\title{
Effect of sustained released metformin therapy on phenotypic and biochemical markers of insulin resistance in polycystic ovary syndrome in South Indian women
}

\author{
Rama Nagendra Kumar ${ }^{1}$, Krishna G. Seshadri ${ }^{1 *}$, Monna Pandurangi ${ }^{2}$
}

\begin{abstract}
${ }^{1}$ Department of Endocrinology, Diabetes and Metabolism, Sri Ramachandra Medical College, Porur, Chennai 600116, Tamil Nadu, India

${ }^{2}$ Department of Reproductive Medicine, Sri Ramachandra Medical College, Porur, Chennai 600116, Tamil Nadu, India
\end{abstract}

Received: 15 December 2015

Revised: 20 February 2016

Accepted: 05 March 2016

\section{*Correspondence:}

Dr. Krishna G. Seshadri,

E-mail: krishnagseshadri@gmail.com

Copyright: () the author(s), publisher and licensee Medip Academy. This is an open-access article distributed under the terms of the Creative Commons Attribution Non-Commercial License, which permits unrestricted non-commercial use, distribution, and reproduction in any medium, provided the original work is properly cited.

\begin{abstract}
Background: Polycystic ovary syndrome (PCOS) is the most common endocrine disorder in young women. Insulin resistance (IR) may play a substantial part in the pathogenesis of PCOS, which leads to type 2 diabetes mellitus (T2DM), cardiovascular disorders and ovarian cancer. Metformin is an insulin sensitizing agent, however its role in PCOS is still controversial.

Methods: Sixty women newly diagnosed with PCOS and healthy age matched controls between 18 to 45 years were enrolled after obtaining informed consent. Women in the PCOS group were started on metformin-SR 1 gram orally, which was then increased to 1.5 grams after two weeks and continued for 6 months. Fasting blood sugar (FBS), fasting insulin (FI), SHBG, TT, free androgen index (FAI), homeostatic model assessment of Insulin resistance (HOMA-IR), homeostatic model assessment of $\beta$ - cell function (HOMA-B), homeostatic model assessment of Insulin sensitivity (HOMA-S) and quantitative insulin sensitivity check index (QUICKI) were measured in the control group as well as PCOS group before and after metformin therapy.

Results: After six months of metformin-SR therapy, PCOS group showed significant reduction in FI, HOMA-IR, HOMA- $\beta$, HOMA-S QUICKI, TT and FAI and significant increase in SHBG levels.

Conclusions: Six months of metformin-SR therapy favorably altered markers of IR, TT, SHBG, anovulation and hyperandrogenism in normoglycemic women with PCOS.
\end{abstract}

Keywords: PCOS, Metformin, Insulin, HOMA, SHBG, Total testosterone

\section{INTRODUCTION}

Polycystic ovary syndrome (PCOS) is a common endocrine disorder of women in the reproductive age with the prevalence of $5-10 \%$ all over the world characterized by chronic anovulation and hyperandrogenism. Even though the aetiology of the syndrome is not fully understood. ${ }^{1}$
Women with PCOS are more prone to T2DM, dyslipidaemia, premature atherosclerosis, and endometrial carcinoma. Insulin resistance (IR) is one of the common factors contributing to the pathogenesis of these abnormalities. The major cause of IR is excess weight and physical inactivity. ${ }^{2}$ Approximately $50-70 \%$ of women with PCOS have IR independent of obesity. ${ }^{3}$ In India the prevalence of IR in PCOS women is about 76.9\%. ${ }^{4}$ HOMA-IR is a surrogate marker; acanthosis 
nigricans (AN) is a phenotypic marker for measuring IR. ${ }^{5,6}$

SHBG is a testosterone binding glycoprotein synthesized in liver. SHBG is reduced in IR and is a sensitive marker for the measurement of IR. Women with PCOS have lower SHBG due to IR irrespective of testosterone levels. ${ }^{7}$ The rise in serum levels of testosterone in PCOS may be due to excess ovarian production of androgens. ${ }^{8}$

Targeting IR by using insulin sensitizing agent such as metformin, used to treat T2DM. In general metformin inhibits hepatic gluconeogenesis, enhances glucose uptake and utilization in peripheral tissues such as skeletal muscle and adipocytes. ${ }^{9,10}$ Metformin may also improve ovulation and reduce circulating androgen levels in PCOS women. ${ }^{11}$

We tried to observe the difference between phenotypic, biochemical and hormonal data in newly diagnosed women with PCOS before and after Met-SR therapy and its impact on IR.

\section{METHODS}

Sixty women newly diagnosed with PCOS, healthy age matched controls between 18-45 years were recruited after obtaining written informed consent from the outpatient clinics of Departments of Endocrinology and Reproductive Medicine at Sri Ramachandra University. The diagnosis of PCOS was based on the Rotterdam /American Society for Reproductive Medicine (ASRM)

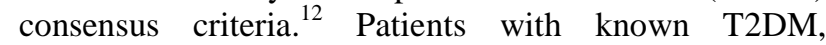
impaired fasting glucose (IFG), impaired glucose tolerance (IGT), thyroid disorders, autoimmune diseases, adrenal, kidney and liver diseases, cardiovascular disease, those planning pregnancy or pregnant, taking oral contraceptive pills (OCP) or metformin, steroidal and non-steroidal anti-inflammatory medications were excluded. This study protocol was approved by the institutional ethics committee of Sri Ramachandra University.

Study subjects were enrolled after baseline measurements' including anthropometry and laboratory parameters from their previous reports, questionnaires regarding the family history of CVD, T2DM, and PCOS was given to each subject. Blood samples from all the subjects were collected in the early follicular phase (between days 3-7) after a 12 hours overnight fasting in EDTA coated and plain vacutainer. Samples were allowed to clot adequately before centrifugation at 2000 RPM (revolutions per minute) for $10 \mathrm{~min}$ and the plasma were separated immediately to estimate the glucose and hormonal levels. The remaining sample was frozen at $80^{\circ} \mathrm{c}$ for further investigations. ${ }^{8}$ Subjects recruited in the PCOS group were treated with $1 \mathrm{gm}$ of Met-SR tablets orally daily for one week which was then titrated to $1.5 \mathrm{gm}$ per day and continued for six months. All parameters were repeated in PCOS subjects after Met-SR therapy. Finally values were compared between the groups.

The study groups were interviewed for a detailed menstrual history including age of menarche, menstrual regularity, duration and number of cycles per year. Oligomenorrhea was defined as inter-menstrual interval of $>35$ days or a total of $<8$ menses per year, AN was defined as hyper pigmented skin over the dorsal surface of the neck and intertriginous area.

\section{Anthropometric measurements}

Anthropometric parameters such as weight, height, waist circumference, hip circumference, waist hip ratio (WHR) and body mass index (BMI) were measured by standard methods.

\section{Hormonal assays}

Plasma glucose was estimated by standard glucose oxidase and peroxidase method (GOD-POD method), and hormones were measured in duplicate by ELISA method by using Bio-RADi Mark Micro plate Reader (S/N 13848), Rayto Micro plate washer RT- $2600 \mathrm{C}$ and GeNei Micro plate Shaker SLM-MPS-250. Insulin was estimated by Cal biotech (catalog No IS130D), Total testosterone (cat. No CAN-TE-250) and SHBG (cat. No CAN-SHBG-4010) were estimated by Diagnostics Biochem Canada ELISA kits.

\section{Insulin resistance assessment}

Insulin resistance was estimated by HOMA-IR method using the formula [HOMAIR = fasting glucose $(\mathrm{mg} / \mathrm{dl}) \mathrm{X}$ fasting insulin $(\mu \mathrm{IU} / \mathrm{ml}) / 405)$. $\quad \beta$-cell function was estimated using HOMA-B by calculating $3.08 \mathrm{X}$ insulin/glucose-3.5. The quantitative insulin sensitivity check index (QUICKI) was used to estimate insulin sensitivity (IS) by calculating $1 /[\log$ fasting insulin $(\mu \mathrm{IU} / \mathrm{ml})+\log$ fasting glucose $(\mathrm{mg} / \mathrm{dl})]$ and HOMA-S was calculated by $146 /$ Insulin $x$ glucose. The free androgen index (FAI) was calculated by total testosterone/SHBG X $100 .^{8,13}$

\section{Statistical analysis}

All results were expressed as mean \pm SD. Statistical analysis was performed using Graph Pad Prism-5. Differences between the control and PCOS groups were analysed by independent t-test and difference with in the PCOS group before and after therapy were measured by paired t-test. P-value of $<0.05$ was considered statistically significant.

\section{RESULTS}

In the base line women with PCOS showed significant higher physical characters such as weight, BMI, WHR. and significant higher insulin indices such as FBS, FI, IR, 
and hyper androgenic characters TT, FAI levels when compared to control group $(\mathrm{P}<0.05)$, lower levels of SHBG and insulin sensitivity markers such as HOMA-S, QUICKI index were observed in PCOS group $(<0.05)$.
There is no significant difference age between the two groups. IR of PCOS group showed significant correlation with BMI and non-significant correlation with SHBG and TT (Table 1 and 2).

Table 1: Anthropometric and Metabolic and Hormonal levels in control and PCOS before and after therapy groups.

\begin{tabular}{|llllll|}
\hline Parameter & Control $(\mathrm{n}=60)$ & PCOS $(\mathrm{n}=60)$ & & P1 \\
\hline Age $($ years $)$ & $26.08 \pm 3.78$ & $24.75 \pm 3.64$ & -------------- & 0.05 & P2 \\
\hline Weight $(\mathrm{kg})$ & $55.45 \pm 7.02$ & $61.95 \pm 11.98$ & $59.05 \pm 10.00$ & $<0.05^{*}$ & $<0.05^{*}$ \\
\hline BMI $\left(\mathrm{Kg} / \mathrm{m}^{2}\right)$ & $23.77 \pm 3.28$ & $25.76 \pm 4.93$ & $24.67 \pm 4.12$ & $<0.05^{*}$ & $<0.05^{*}$ \\
\hline WHR & $0.78 \pm 0.04$ & $0.81 \pm 0.05$ & $0.79 \pm 0.05$ & $<0.05^{*}$ & $<0.05^{*}$ \\
\hline FBS $(\mathrm{mg} / \mathrm{dl})$ & $87.63 \pm 10.11$ & $94.73 \pm 6.80$ & $86.43 \pm 6.93$ & $<0.05^{*}$ & $<0.05^{*}$ \\
\hline FI $(\mu \mathrm{IU} / \mathrm{ml})$ & $10.65 \pm 2.48$ & $14.28 \pm 1.47$ & $11.62 \pm 1.55$ & $<0.05^{*}$ & $<0.05^{*}$ \\
\hline HOMA-IR & $1.36 \pm 0.32$ & $1.85 \pm 0.99$ & $1.48 \pm 0.20$ & $<0.05^{*}$ & $<0.05^{*}$ \\
\hline HOMA- $\beta$ & $128.05 \pm 31.13$ & $132.68 \pm 19.24$ & $137.87 \pm 23.35$ & $0.32 \mathrm{~ns}^{*}<0.05^{*}$ \\
\hline HOMA-S & $77.92 \pm 19.08$ & $54.50 \pm 5.93$ & $68.74 \pm 9.55$ & $<0.05^{*}$ & $<0.05^{*}$ \\
\hline QUICKI & $0.33 \pm 0.01$ & $0.31 \pm 0.01$ & $0.33 \pm 0.01$ & $<0.05^{*}$ & $<0.05^{*}$ \\
\hline TT (nmol/l) & $1.77 \pm 0.51$ & $2.65 \pm 0.74$ & $2.44 \pm 0.73$ & $<0.05^{*}$ & $<0.05^{*}$ \\
\hline SHBG $(\mathrm{nmmol} / \mathrm{l})$ & $69.79 \pm 5.39$ & $51.84 \pm 12.25$ & $59.17 \pm 11.94$ & $<0.05^{*}$ & $<0.05^{*}$ \\
\hline FAI & $2.56 \pm 0.11$ & $5.19 \pm 1.15$ & $4.15 \pm 0.96$ & $<0.05^{*}$ & $<0.05^{*}$ \\
\hline Oligomenorrhoea & 0 & 41 & 21 & $<0.05^{*}$ & $<0.05^{*}$ \\
\hline Acanthosis nigricans & 0 & 26 & 12 & $<0.05^{*}$ & $<0.05^{*}$ \\
\hline
\end{tabular}

Data were expressed in Mean \pm SD by using t-test; clinical indices were done by Chi-squire test. PCOS BT $=$ PCOS before therapy; PCOS AT= PCOS after therapy; P1 = P-value between control and PCOS BT groups; P2 = P-value between PCOS BT and PCOS AT; $*$ = statistically significant $(\mathrm{P}<0.05), \mathrm{ns}=$ statistically non-significant $(\mathrm{P}>0.05)$.

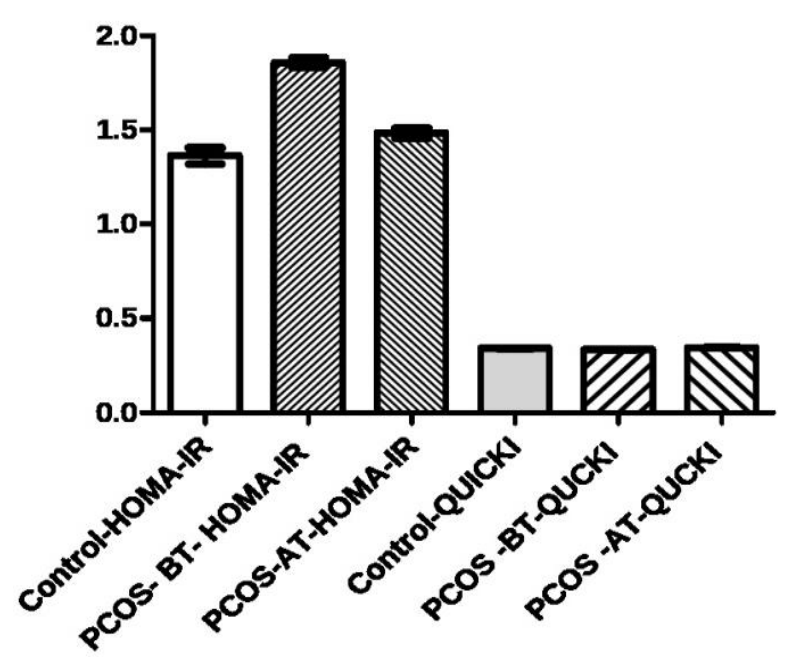

Data were expressed in Mean \pm SD, PCOS BT $=$ PCOS before therapy; PCOS AT $=$ PCOS after therapy HOMA-IR = Homeostatic Model Assessment of Insulin Resistance; QUICKI= Quantitative Insulin Sensitivity Index

Figure 1a: Insulin resistance parameters of the control and PCOS groups.

After six months of Met-SR therapy the PCOS group showed normalization of menstrual cycle and decrease in
AN and showed significant reduction in weight, BMI, WHR, FBS, FI, HOMA-IR, TT and FAI was a significant increase in $\beta$-cell function, Insulin sensitivity index and SHBG levels. There was correlation of BMI, SHBG and TT with HOMA-IR (Table 1, 2 \& Figure 1a, 1b, 1c).

Table 2: Correlation between BMI and hormonal levels with insulin resistance.

\begin{tabular}{|c|c|c|c|c|}
\hline \multirow{3}{*}{ Parameters } & \multicolumn{4}{|c|}{ PCOS group $(n=60)$} \\
\hline & \multicolumn{2}{|c|}{ Before therapy } & \multicolumn{2}{|c|}{ After therapy } \\
\hline & $\begin{array}{l}\text { r- } \\
\text { value }\end{array}$ & $\begin{array}{l}\text { p- } \\
\text { value }\end{array}$ & $\begin{array}{l}\text { r- } \\
\text { value }\end{array}$ & $\begin{array}{l}\text { p- } \\
\text { value }\end{array}$ \\
\hline $\begin{array}{l}\text { HOMA-IR } \\
\text { vs BMI }\end{array}$ & 0.29 & $<0.05^{*}$ & 0.28 & $<0.05^{*}$ \\
\hline $\begin{array}{l}\text { HOMA-IR } \\
\text { vs SHBG }\end{array}$ & 0.13 & $0.30^{\mathrm{ns}}$ & 0.26 & $<0.05^{*}$ \\
\hline $\begin{array}{l}\text { HOMA- IR } \\
\text { vs TT }\end{array}$ & 0.17 & $0.17^{\text {ns }}$ & 0.24 & $<0.05 *$ \\
\hline
\end{tabular}

All the values were expressed by Pearson's correlation. ${ }^{*}=$ statistically significant $(\mathrm{P}<0.05)$, $\mathrm{ns}=$ statistically nonsignificant $(\mathrm{P}>0.05)$. 


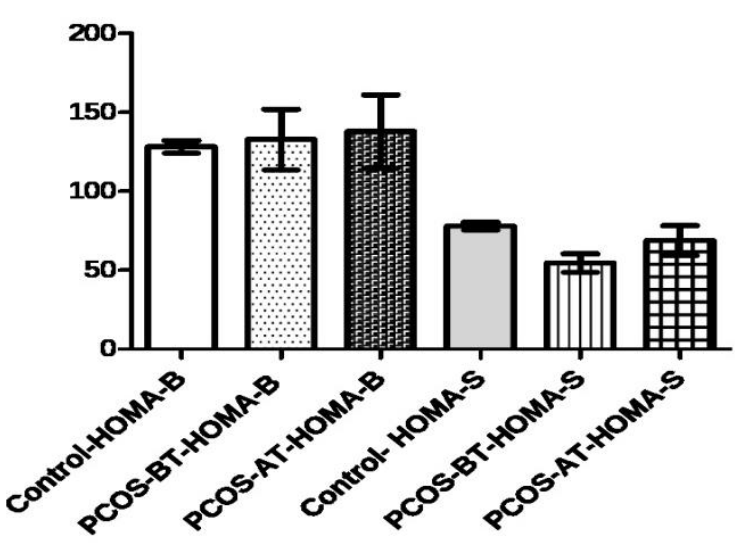

Data were expressed in Mean $\pm \mathrm{SD}$, PCOS BT $=$ PCOS before therapy; PCOS AT $=$ PCOS after therapy HOMA-B = Homeostatic Model Assessment of $\beta$-cell function; HOMA-S = Homeostatic Model Assessment of Insulin Sensitivity

Figure 1b: Insulin Sensitivity and $\beta$-cell parameters of the control and PCOS groups.

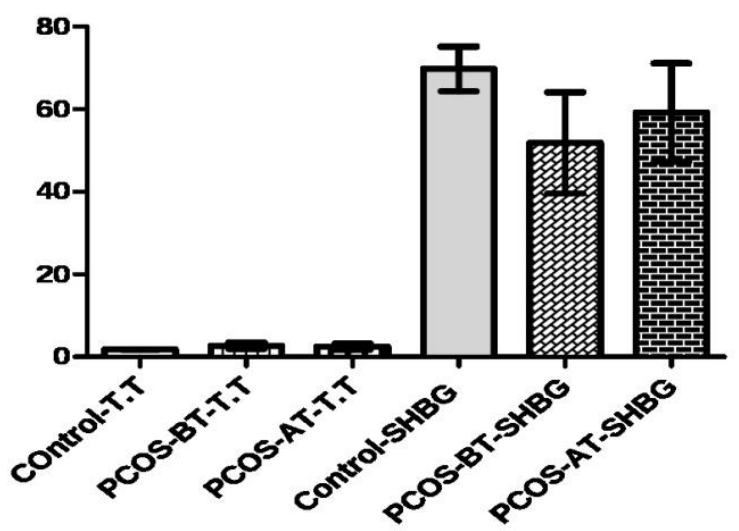

Data were expressed in Mean $\pm \mathrm{SD}$, PCOS BT $=$ PCOS before therapy; PCOS AT $=$ PCOS after therapy $\mathrm{TT}=$ Total testosterone; $\mathrm{SHBG}=$ Sex hormone binding globulin.

Figure 1c: Hyper androgenic parameters of the control and PCOS groups.

\section{DISCUSSION}

To our knowledge, this is the first study to report all parameters of insulin dynamics in the south Asian population with PCOS and the effect of Met-SR on these parameters. PCOS women were more obese (BMI of $25.75 \pm 4.93)$ when compared to controls $(23.77 \pm 3.28)$. Met-SR therapy was significantly decreased BMI in PCOS women (from $25.75 \pm 4.93$ to $24.67 \pm 4.12$ ), which was similar to others. ${ }^{5}$

Met-SR therapy also reduced TT levels significantly in agreement with the previous studies. ${ }^{14,15}$ It also improved menstrual abnormalities in women with PCOS, which was similar to other studies. ${ }^{16,17}$ Metformin may reduce the severity of AN, In the present study we confirmed that six months of Met-SR therapy had enough to treat $\mathrm{AN}$ in PCOS women.
We have also demonstrated that metformin-SR therapy the PCOS group showed significant decrease in HOMAIR, which is similar to the previous studies. ${ }^{18} \mathrm{We}$ also observed the improvement in $\beta$-cell function. This may be due to reduction in IR. Met-SR therapy also showed reduction testosterone, increase SHBG levels, ovulatory frequency and menstrual irregularities, which is similar to other studies. $^{19,20}$

In the present study we have confirmed that, women with PCOS showed correlation between IR and BMI before and after therapy, and also observed a positive correlation between SHBG and TT with HOMA-IR after six months of Met-SR therapy.

\section{CONCLUSIONS}

Six months of Met-SR therapy seems to be effective in decreasing BMI, FBS, FI IR, SHBG and FAI. All function together these may lead to improvement in the menstrual irregularities and hyperandrogenism in normoglycemic PCOS women.

\section{ACKNOWLEDGEMENTS}

We would like to specially thank Dr. Usha Rani, Dr. Sanjeeva Reddy from Reproductive Medicine SRMC for supporting in sample collection and Dr. Ravi Shankar from the community medicine SRMC for helping in statistics and Dr. Sriram Mahadevan and Dr. Nithya Ramalingam from the Department of Endocrinology, Diabetes and Metabolism for supporting in writing this paper.

Funding: We would like to thank Bristol- Myers Squibb for partial funding of this project.

Conflict of interest: None declared

Ethical approval: The study was approved by Institutional ethics committee of Sri Ramachandra University, Porur, Chennai-600116.

\section{REFERENCES}

1. Asmathulla S, RupaVani K, Kripa S, Rajarajeswari R. Insulin resistance and itsrelation to inflammatory status and serum lipids among young women with PCOS. Int J Reprod Contracept Obstet Gynecol. 2013;2:325-9.

2. Ramanand SJ, Ghongane BB, Ramanand JB, Patwardhan MH, Ghanghas RR, Jain SS. Clinical characteristics of polycystic ovary syndrome in Indian women. Indian $\mathrm{J}$ Endo crinol Metab. 2013;17:138-45.

3. Mukherjee S, Maitra A. Molecular and genetic factors contributing to insulin resistance in polycystic ovary syndrome. Indian J Med Res. 2010;131:74360.

4. Kalra A, Nair S, Rai L. Association of obesity and insulin resistance with dyslipidemia in Indian women 
with polycystic ovarian syndrome. Indian J Med Sci. 2006;60:447-53.

5. Velija-Asimi Z. Evaluation of endocrine changes in women with the polycystic ovary syndrome during metformin treatment. Bosn J Basic Med Sci 2013;13:185-5.

6. Fleming R, Hopkinson ZE, Wallace AM, Greer IA, Sattar N. Ovarian function and metabolic factors in women with oligomenorrhea treated with metformin in a randomized double blind placebo-controlled trial. J Clin Endocrinol Metab. 2002;87:569-74.

7. Chen C, Smothers J, Lange A, Nestler JE, Strauss Iii JF, Wickham Iii EP. Sex hormone-binding globulin genetic variation: associations with type 2 diabetes mellitus and polycystic ovary syndrome. Minerva Endocrinol. 2010;35:271-80.

8. Panidis D, Tziomalos K, Papadakis E, Chatzis P, Kandaraki EA, Tsourdi EA. Associations of menstrual cycle irregularities with age, obesity and phenotype in patients with polycystic ovary syndrome. Hormones. 2015;14:431-7.

9. Del Barco S, Vazquez-Martin A, Cufi S, OliverasFerraros C, Bosch-Barrera J, Joven J. Metformin: multi-faceted protection against cancer, Onco target. 2011;2:896-917.

10. Hwang KR, Choi YM, Kim JJ, Chae SJ, Park KE, Jeon HW, et al. Effects of insulin-sensitizing agents and insulin resistance in women with polycystic ovary syndrome. Clin Exp Reprod Med. 2013;40:100-5.

11. Palomba S, Falbo A, Zullo F, Orio F. Evidencebased and potential benefits of metformin in the polycystic ovary syndrome: a comprehensive review. Endocr Rev. 2009;30:1-50.

12. Rotterdam ESHRE/ASRM-sponsored PCOS consensus workshop group. Revised 2003 consensus on diagnostic criteria and long-term health risks related to polycystic ovary syndrome (PCOS). Hum Reprod. 2004;19:41-7.

13. Mohiyiddeen L, Watson AJ, Apostolopoulos NV, Berry R, Alexandraki KI, Jude EB. Effects of low dose metformin and rosiglitazone on biochemical, clinical, metabolic and biophysical outcomes in polycystic ovary syndrome. J Obstet Gynaecol. 2013;33:165-70.
14. Santana LF, de Sa MF, Ferriani RA, de Moura MD, Foss MC, dos Reis R M. Effect of metformin on the clinical and metabolic assessment of women with polycystic ovary syndrome. Gynecol Endocrinol. 2004;19:88-96.

15. Leanza V, Coco L, Grasso F, Leanza G, Zarbo G, Palumbo M. Ovulation induction with clomiphene citrate and metformin in women with polycystic ovary syndrome. Minerva Ginecol. 2014;66:299-301.

16. Glueck CJ, Wang P, Fontaine R, Tracy T, SieveSmith L. Metformin induced resumption of normal menses in 39 of $43(91 \%)$ previously amenorrheic women with the polycystic ovary syndrome. Metabolism. 1999;48:511-9.

17. Moghetti P, Castello R, Negri C, Tosi F, Perrone F, Caputo M. Metformin effects on clinical features, endocrine and metabolic profiles, and insulin sensitivity in polycystic ovary syndrome: a randomized, double- blind, placebo-controlled 6month trial, followed by open, long-term clinical evaluation. J Clin Endocrinol Metab. 2000;85:13946.

18. Elkind-Hirsch K, Marrioneaux O, Bhushan M, Vernor D, Bhushan R. Comparison of Single and combined treatment with exenatide and metformin on menstrual cyclicity In overweight women with polycystic ovary syndrome. J Clin Endocrinol Metab. 2008;93:2670-78.

19. Palomba S, Falbo A, Russo T, Orio F, Tolino A, Zullo F. Systemic and local effects of metformin administration in patients with polycystic ovary syndrome (PCOS): relationship to the ovulatory response, Hum Reprod. 2010;25:1005-13.

20. Madnani N, Khan K, Chauhan P, Parmar G. Polycystic ovarian syndrome. Indian J Dermatol Venereol Leprol. 2013;79:310-21.

Cite this article as: Kumar RN, Seshadri KG, Pandurangi M. Effect of sustained released metformin therapy on phenotypic and biochemical markers of insulin resistance in polycystic ovary syndrome in South Indian women. Int J Reprod Contracept Obstet Gynecol 2016;5:1026-30. 